2008

\title{
Collective behavior of stabilized reaction-diffusion waves
}

Aaron J. Steele

Mark Tinsley

Kenneth Showalter

Follow this and additional works at: https://researchrepository.wvu.edu/faculty_publications

\section{Digital Commons Citation}

Steele, Aaron J.; Tinsley, Mark; and Showalter, Kenneth, "Collective behavior of stabilized reaction-diffusion waves" (2008). Faculty Scholarship. 87.

https://researchrepository.wvu.edu/faculty_publications/87

This Article is brought to you for free and open access by The Research Repository @ WVU. It has been accepted for inclusion in Faculty Scholarship by an authorized administrator of The Research Repository @ WVU. For more information, please contact ian.harmon@mail.wvu.edu. 


\title{
Collective behavior of stabilized reaction-diffusion waves
}

\author{
Aaron J. Steele, Mark Tinsley, and Kenneth Showalter \\ Department of Chemistry, West Virginia University, Morgantown, West Virginia 26506-6045, USA
}

(Received 31 January 2008; accepted 29 February 2008; published online 27 June 2008)

\begin{abstract}
Stabilized wave segments in the photosensitive Belousov-Zhabotinsky reaction are directionally controlled with intensity gradients in the applied illumination. The constant-velocity waves behave like self-propelled particles, and multiple waves interact via an applied interaction potential. Alignment arises from the intrinsic properties of the interacting waves, leading to processional and rotational behavior. (c) 2008 American Institute of Physics. [DOI: 10.1063/1.2900386]
\end{abstract}

\begin{abstract}
Self-organization in far-from-equilibrium systems arises from local feedback processes that result in larger scale order. Examples include spatiotemporal patterns in chemical systems ${ }^{1-3}$ and in granular media. ${ }^{4-6}$ Similar self-organization principles are thought to account for the collective behavior seen in biological systems, such as patterns in bacteria colonies, schools of fish, and flocks of birds. $^{7,8}$ Here, the large-scale order of the group arises from local interactions between members of the group. Field studies of collective behavior in groups of living organisms have inspired theoretical modeling studies of populations of interacting agents. These studies have offered valuable insights into group behavior, identifying key features such as density dependent alignment in swarms of locusts and response behavior to food or threat in schools of fish. ${ }^{9,10}$ The specific biological traits that give rise to the interaction rules in different species remain to be elucidated in many cases. ${ }^{10}$ In this paper, we present an experimental system for studying collective behavior arising from interactions of individuals within a group. The group members consist of stabilized chemical waves that behave like interacting self-propelled particles.
\end{abstract}

\section{INTRODUCTION}

The stabilized waves are studied in a thin gel that is loaded with the catalyst of the photosensitive BelousovZhabotinsky (BZ) reaction, ${ }^{11,12}$ which is immersed in continually refreshed catalyst-free BZ reaction mixture. The particle-like waves can be directionally controlled by imposing gradients of light intensity, giving rise to excitability gradients. ${ }^{13}$ A computer controlled feedback algorithm for monitoring the waves and applying the intensity gradients allows the construction of interaction potentials between the waves based upon their mutual locations.

We first describe the propagation behavior of single waves in static excitability landscapes. The behavior of single waves provides a basis for understanding interacting waves, where the excitability landscape is dynamically varied according to the presence of neighboring waves. Our photosensitive chemical system of interacting waves lies between that of a naturally occurring system with inherent interaction properties and a theoretical system with model dependent properties. We describe emergent ordered behavior, including processional and rotational modes as well as more complex behavior. The collective behavior arises from a combination of the inherent properties of stabilized waves in excitability gradients and the interactions between the waves due to the applied interaction potential.

\section{METHODS}

Experiments were carried out with the photosensitive BZ reaction, ${ }^{11,12}$ which was monitored with a computer interfaced camera and illuminated with a computer controlled video projector. The wave behavior was studied in a thin layer of silica gel in which the ruthenium (II)-bipyridil catalyst was immobilized. The gel was cast onto a microscope slide and bathed in continually refreshed, catalyst-free BZ solution containing $0.166 \mathrm{M} \mathrm{NaBrO}_{3}, 0.054 \mathrm{M}$ malonic acid, $0.162 \mathrm{M}$ bromomalonic acid, and $0.366 \mathrm{M} \mathrm{H}_{2} \mathrm{SO}_{4}$.

Simulations of the spatiotemporal behavior were carried out with a two-variable Oregonator model for the photosensitive $\mathrm{BZ}$ reaction, ${ }^{14,15}$

$$
\frac{\partial u}{\partial t}=\frac{1}{\epsilon}\left\{u-u^{2}-[\phi(x, y)+f v] \frac{u-q}{u+q}\right\}+D_{u} \nabla^{2} u,
$$

$$
\frac{\partial v}{\partial t}=u-v
$$

where $u$ and $v$ are the dimensionless concentrations of $\mathrm{HBrO}_{2}$ and $\mathrm{Ru}(\mathrm{bpy})_{3}^{3+}, D_{u}$ is the diffusion coefficient of $\mathrm{HBrO}_{2}$, and $\phi$ represents the rate of bromide production due to the irradiation. The Euler method was used in the numerical simulations, with $d x=0.15$ and $d t=0.001$, and the parameters were $f=1.4, q=0.0002, \epsilon=0.01$, and $D_{u}=1.0$.

For stabilization and directional control, two feedback algorithms are applied to each propagating wave. The first is a proportional-integral-derivative (PID) algorithm ${ }^{16}$ used to stabilize the wave segment, which maintains a constant wave size ${ }^{17-19}$

$$
\phi_{f}=g\left[S+p_{i} \int_{t^{\prime}=t-t_{0}}^{t^{\prime}=t} S\left(t^{\prime}\right) d t^{\prime}-p_{d}\left(\frac{d S}{d t}\right)\right]+\phi_{0},
$$

where $\phi_{f}$ is the applied illumination intensity, $\phi_{0}$ is the offset or background illumination intensity, $S$ is the wave size, and $g$ is the gain. The respective contributions of the integral and derivative terms are $p_{i}$ and $p_{d}{ }^{16}$ The second is an excitability 
(a)

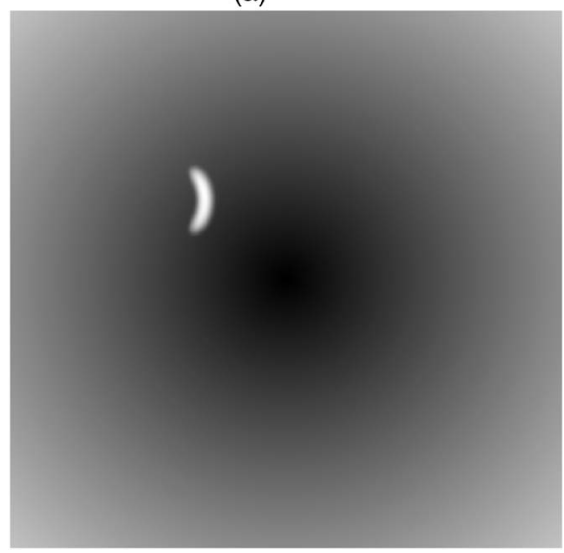

(b)



(c)

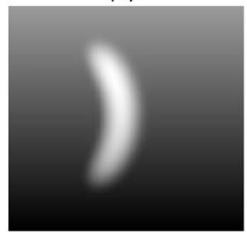

FIG. 1. (a) A stabilized wave propagating in a radially symmetric excitability potential. Control box for sampling the potential (b) and control box for applying the light intensity gradient (c), which is the component of the potential perpendicular to the wave velocity. Panels (b) and (c) show enlargement of a wave in (a) and corresponding control box. The intensity gradient corresponding to the potential is shown in (b), and the intensity gradient applied to control the wave is shown in (c).

gradient arising from the imposed illumination, which is computed from a potential function $U(\mathbf{r})$. The gradient of the potential is evaluated at the centroid of the wave, ${ }^{20}$ and the resulting illumination is computed from the component perpendicular to the wave velocity $\mathbf{v}$, as shown in Fig. 1. The intensity $\phi_{i j}$ at point $\mathbf{r}_{i, j}$ inside a small control box around the wave is determined by

$$
\phi_{i j}=\phi_{f}+\left(\hat{\mathbf{v}}_{\perp} \cdot \nabla U\right)\left[\hat{\mathbf{v}}_{\perp} \cdot\left(\mathbf{r}_{i j}-\mathbf{r}_{c}\right)\right],
$$

where $\mathbf{r}_{c}$ is the centroid of the wave and $\phi_{f}$ is the wave stabilization intensity. We apply the component of the potential perpendicular to the wave velocity, which provides directional control of a wave by creating an effective turning force. This is equivalent to a rotational force acting on a point object; however, the two-dimensional structure of the wave leads to asymmetric responses in the turning rate, as discussed in Sec. V.

\section{EXCITABILITY POTENTIALS}

The behavior of single waves propagating in various excitability potentials has been investigated. The propagation behavior in four different potentials is shown in Fig. 2. We find that the wave generally approaches a path of minimum potential, where $\nabla U=0$. For the harmonic valley potential in (a), (b) and the radially symmetric Lennard-Jones-type potential in $(\mathrm{g}),(\mathrm{h})$, these paths are in the $x$ direction and around a center-oriented circle, respectively. For the radially symmetric quadratic potential shown in (c), (d), there is only a single point minimum, and the constant velocity wave therefore traces out a sequence of approximately periodic loops that pass near the minimum. Quasiperiodic-type orbits are exhibited in other radially symmetric potentials, such as a cubic potential. As the wave approaches the minimum, it turns to align with a radial along which $\hat{\mathbf{v}}_{\perp} \cdot \nabla U=0$. As it travels away from the minimum close to the radial, the wave eventually turns again toward the minimum. There is no
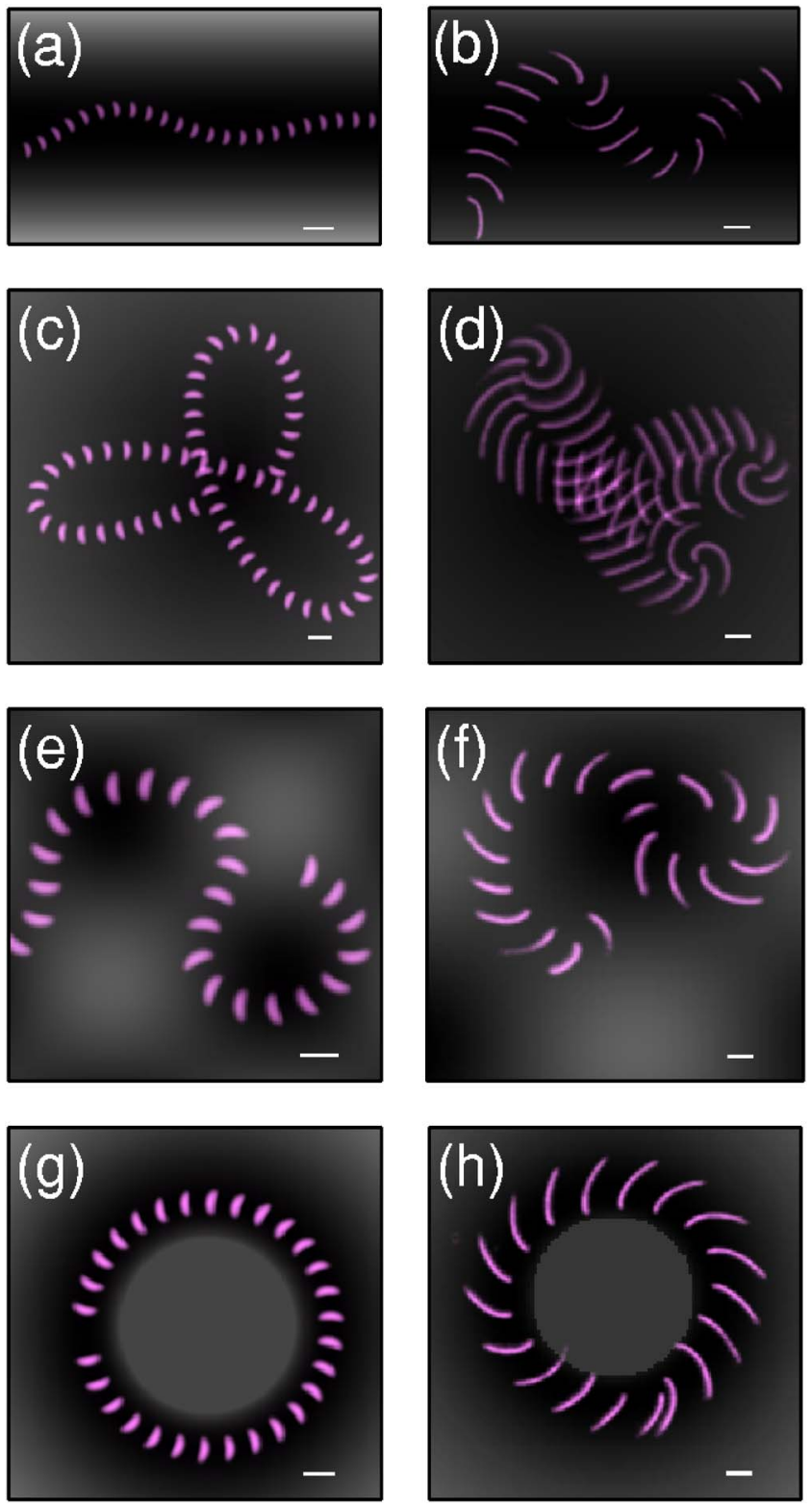

FIG. 2. (Color online) The left and right panels show overlaid images from simulations and experiments of waves propagating in excitability potentials, which are superimposed in gray scale. (a), (b) A wave propagating in a harmonic valley potential, $\quad U(y)=\alpha y^{2}, \quad \alpha_{\text {sim }}=3.6 \times 10^{-7}, \quad \alpha_{\exp }$ $=0.21 \mathrm{~mW} \mathrm{~cm}{ }^{-2} / \mathrm{mm}^{2}$. The gray level of the potential has been scaled for the image. (c), (d) A wave propagating in a radially symmetric quadratic potential, $U(r)=\alpha r^{2}, \alpha_{\text {sim }}=1.1 \times 10^{-7}, \alpha_{\text {exp }}=0.29 \mathrm{~mW} \mathrm{~cm} \mathrm{~cm}^{-2} / \mathrm{mm}^{2}$. (e), (f) A wave propagating in a two-dimensional, sine-wave potential, $U(x, y)$ $=\alpha[\sin (x \pi / \beta) \sin (y \pi / \beta)], \alpha_{\text {sim }}=4.5 \times 10^{-2}, \beta_{\text {sim }}=16, \alpha_{\text {exp }}=0.83 \mathrm{~mW} \mathrm{~cm}^{-2}$, $\beta_{\text {exp }}=9.1 \mathrm{~mm}$. (g), (h) A wave propagating in a Lennard-Jones-type potential described by Eq. (5), with $a_{\text {sim }}=1.13, b_{\text {sim }}=0.15, \quad c_{\text {sim }}=5$, and $a_{\exp }$ $=0.11 \mathrm{~mm}^{2}, b_{\exp }=8.4 \times 10^{-2} \mathrm{~mm}, c_{\exp }=108 \mathrm{~mW} \mathrm{~cm}^{-2}$. (a) - (h) Frame inter$\mathrm{val}$ is $70 \mathrm{~s}$ in the experiments and 1.0 in the simulations. The scale bar is $1.5 \mathrm{~mm}$ in the experiments and 3.75 in the simulations. Parameters constant throughout the paper: (experiments) $g=2 \mathrm{~mW} \mathrm{~cm}^{-2} / \mathrm{mm}, p_{i}=0.05 / \tau_{0}$, and $p_{d}=0.8 \tau_{0}$, where $\tau_{0}=2 \mathrm{~s}$; (simulations) $g=0.83, p_{i}=0$, and $p_{d}=0$.

simple path of minimum potential in the sine-wave excitability landscape of hills and valleys shown in (e), (f), but rather paths of relative minimum potential, where a wave finds its way from one valley to another along the minimum between two hills. Long transients are occasionally exhibited in this complex excitability landscape; however, the wave trajectory 




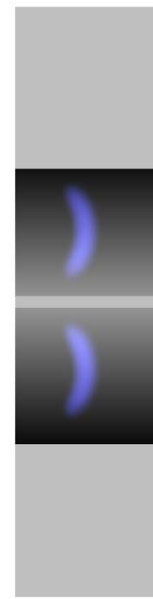

(b)

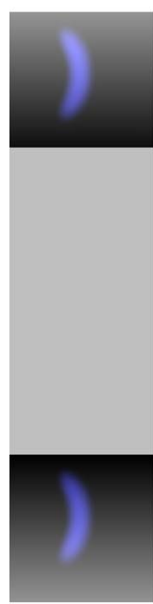

(c)
FIG. 3. (Color online) (a) LJ-type potential with an equilibrium distance $r_{0}=15$. The potential function determines the intensity gradients applied to the interacting waves, which affect the direction but not the magnitude of the wave velocities. Waves with applied intensity gradients, (b) where the inter-wave distance is less than the equilibrium distance, $|\mathbf{r}|=7$, and (c) where the inter-wave distance is greater than the equilibrium distance, $|\mathbf{r}|=22$. The light gray region outside the control boxes corresponds to the background intensity.

eventually follows a path near the relative minimum potential.

\section{INTERACTING WAVES}

We use a Lennard-Jones (LJ) type potential as the basis for interactions between two or more waves,

$$
U_{m, n}=c\left(\frac{a}{\left|\mathbf{r}_{n}-\mathbf{r}_{m}\right|^{2}}-\frac{b}{\left|\mathbf{r}_{n}-\mathbf{r}_{m}\right|}\right),
$$

where $\mathbf{r}_{m}$ is the location of the centroid of wave $m$, which experiences the potential $U_{m, n}$ due to the presence of wave $n$ at location $\mathbf{r}_{n}$. The constant $c$ determines the wave interaction strength, and the constants $a$ and $b$ determine the equilibrium distance $r_{0}=2 a / b$, where $\nabla U_{m, n}=0$. The interaction potential is illustrated in Fig. 3(a).

When the wave separation is less than the equilibrium distance, $|\mathbf{r}|<r_{0}$, the intensity gradients applied to the two interacting waves cause them to turn away from each other, Fig. 3(b). When the wave separation is greater than the equilibrium distance, $|\mathbf{r}|>r_{0}$, the intensity gradients cause the waves to turn toward each other, Fig. 3(c). Figures 3(b) and 3(c) illustrate how the waves experience a mutually repulsive potential when the wave separation is less than the equilibrium distance and a mutually attractive potential when the wave separation is greater than the equilibrium distance. In the general $N$-wave case, the light intensity applied to wave $m$ is determined by the sum of the potential contributions,

$$
U_{\text {sum }}=\sum_{n=1, n \neq m}^{N} U_{m, n} .
$$

We now describe the varieties of behavior in our simulations and experiments. The various types of behavior are
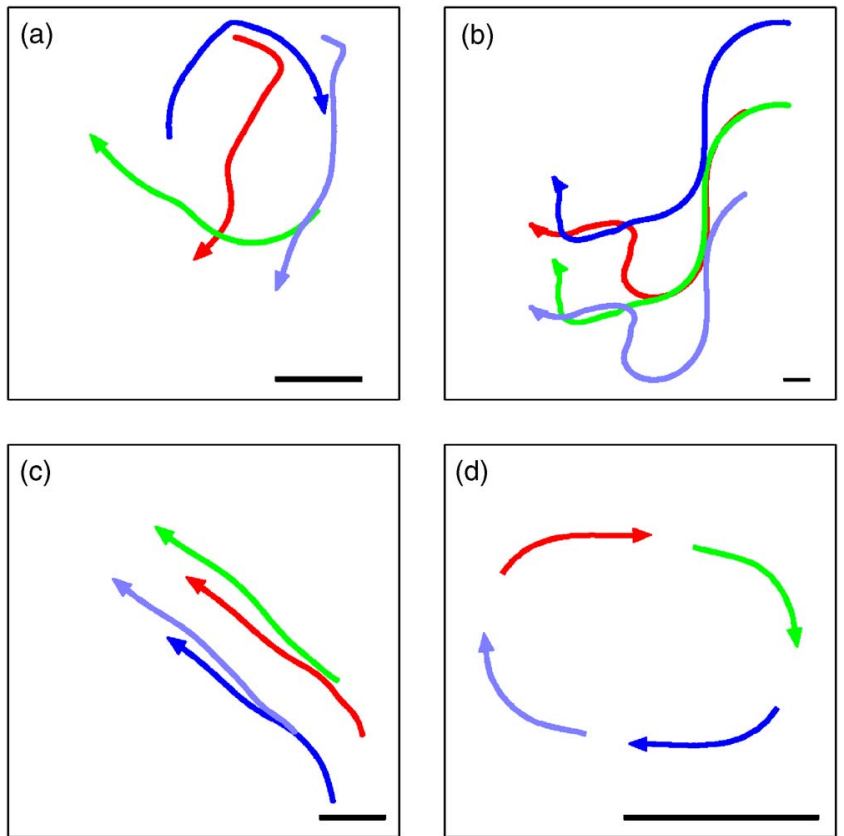

FIG. 4. (Color online) Wave trajectories from simulations demonstrating possible types of motion. The particular behavior depends on the interaction strength, the number of waves, and the initial conditions. As the strength of the wave interactions is increased by increasing the value of $c$, the following types of motion are exhibited: For low values of the interaction strength $c$, the motion is noncohesive (a), $c_{\text {sim }}=5.1$. As the value of $c$ is increased, wandering behavior may be exhibited (b), $c_{\text {sim }}=6.0$. Parallel motion (c), $c_{\text {sim }}=30$, is possible over a wide range of interaction strengths. With high values of $c$, rotational modes become possible (d), $c_{\text {sim }}=70$. Panel (d) shows the rigid rotation of four waves; other rotational modes are shown in Figs. 7 and 8. Other parameters are as in Fig. 5. The scale bar at the bottom of each panel is equal to the length $r_{0}$, which is 15 in each of the simulations. The other LJ parameters are $a_{\text {sim }}=1.13, b_{\text {sim }}=0.15$.

illustrated in Fig. 4, where the calculated wave trajectories are indicated by a line with an arrow. The specific motion depends on the interaction strength, the number of waves, and the initial conditions.

\section{A. Processional behavior}

The most common type of behavior observed is processional behavior, illustrated in Fig. 5 for two, three, and four waves. The waves organize into traveling packs from random initial conditions, with mutually aligned velocities. In a finite medium, the waves reflect at the boundaries and then reorganize into a similar processional structure. ${ }^{21}$ Simulations conducted with an infinite medium demonstrate that the processional structures are maintained indefinitely, with the group centroid traveling in a straight line or a very gradual arc. Size limitations in the experimental system prevented the investigation of groups of more than four or five waves; however, simulations of groups of up to 20 waves have been conducted. Processional behavior is found over a wide range of interaction strengths and initial conditions in the experiments and simulations.

Alignment arises with wave trajectories following paths of minimum potential, along which $\hat{\mathbf{v}}_{\perp} \cdot \nabla U=0$. A wave in a processional group travels along such a path without experiencing an excitability gradient. If minimum potential paths 

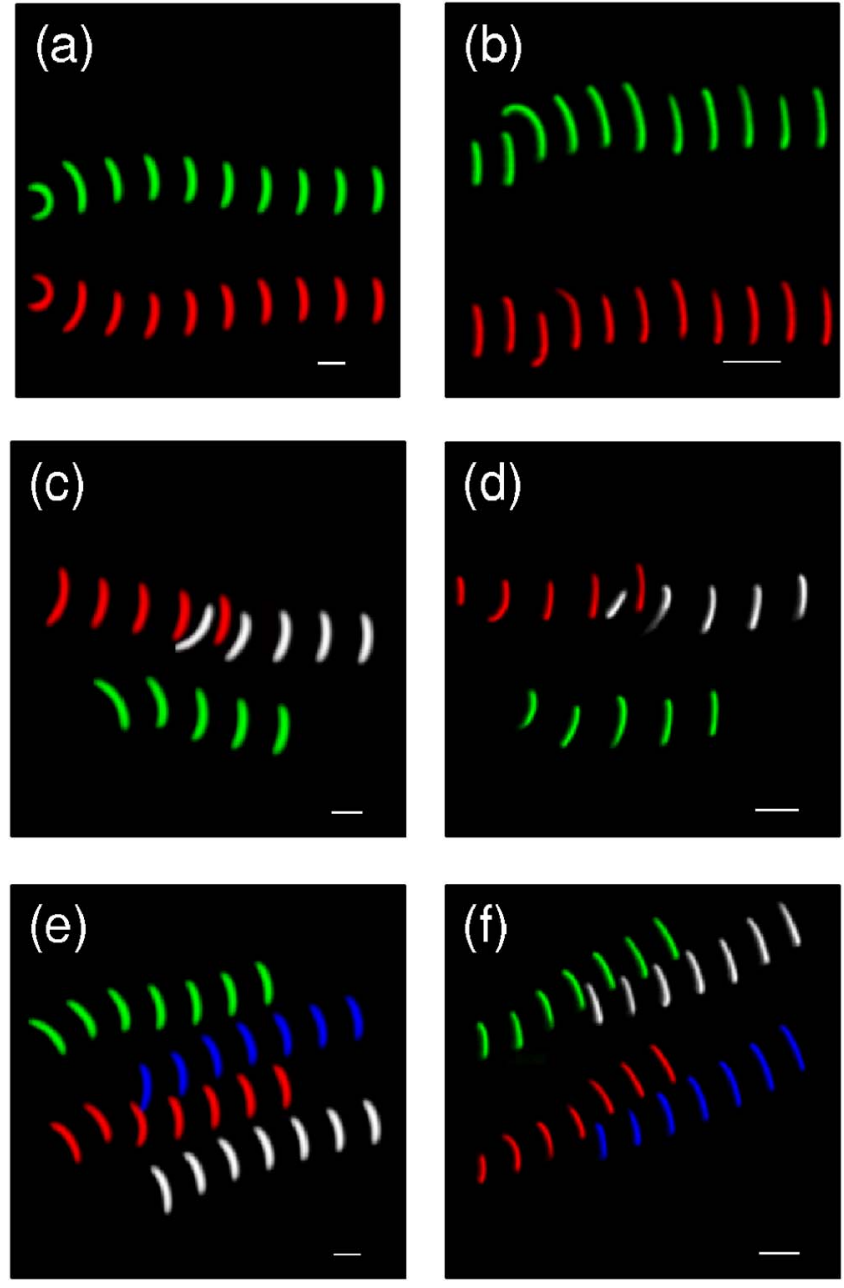

FIG. 5. (Color online) The left and right panels show a series of superimposed images of processional waves in simulations and experiments, respectively. The LJ interaction parameters are $a_{\text {sim }}=1.13, b_{\text {sim }}=0.15, a_{\exp }$ $=0.21 \mathrm{~mm}^{2}$, and $b_{\exp }=8.4 \times 10^{2} \mathrm{~mm}$. In panels (a) and (b), two waves initially closer than the equilibrium distance move apart and then maintain alignment, with $c_{\text {sim }}=5.0$ and $c_{\text {exp }}=3.3 \times 10^{3} \mathrm{~mW} \mathrm{~cm}^{-2}$. In panels (c) and (d), three waves move in parallel, with $c_{\text {sim }}=8.0$ and $c_{\exp }=8.3$ $\times 10^{2} \mathrm{~mW} \mathrm{~cm}^{-2}$. In panels (e) and (f), four waves move in parallel, with $c_{\text {sim }}=8.0$ and $c_{\text {exp }}=8.3 \times 10^{2} \mathrm{~mW} \mathrm{~cm}^{-2}$. Time between frames: $\sim 100 \mathrm{~s}$ in the experiments and 1.5 in the simulations. The length scale bar at the bottom of each panel is equal to $r_{0} / 4$.

simultaneously exist for every wave of a group, the corresponding pattern of waves is then a steady state configuration. Small perturbations of the steady state configurations are followed by a rapid decay back to similar processional configurations.

\section{B. Rotational behavior}

Rotational modes also occur but are less common. Figure 6 shows rotational behavior in experiments and simulations for two, three and four waves. The rotations develop at high values of the interaction parameter $c$ and with initial conditions of waves equally spaced around a ring. The stability of these simple rotational modes has been examined in simulations. Figures 7(a)-7(c) show an unstable two-wave
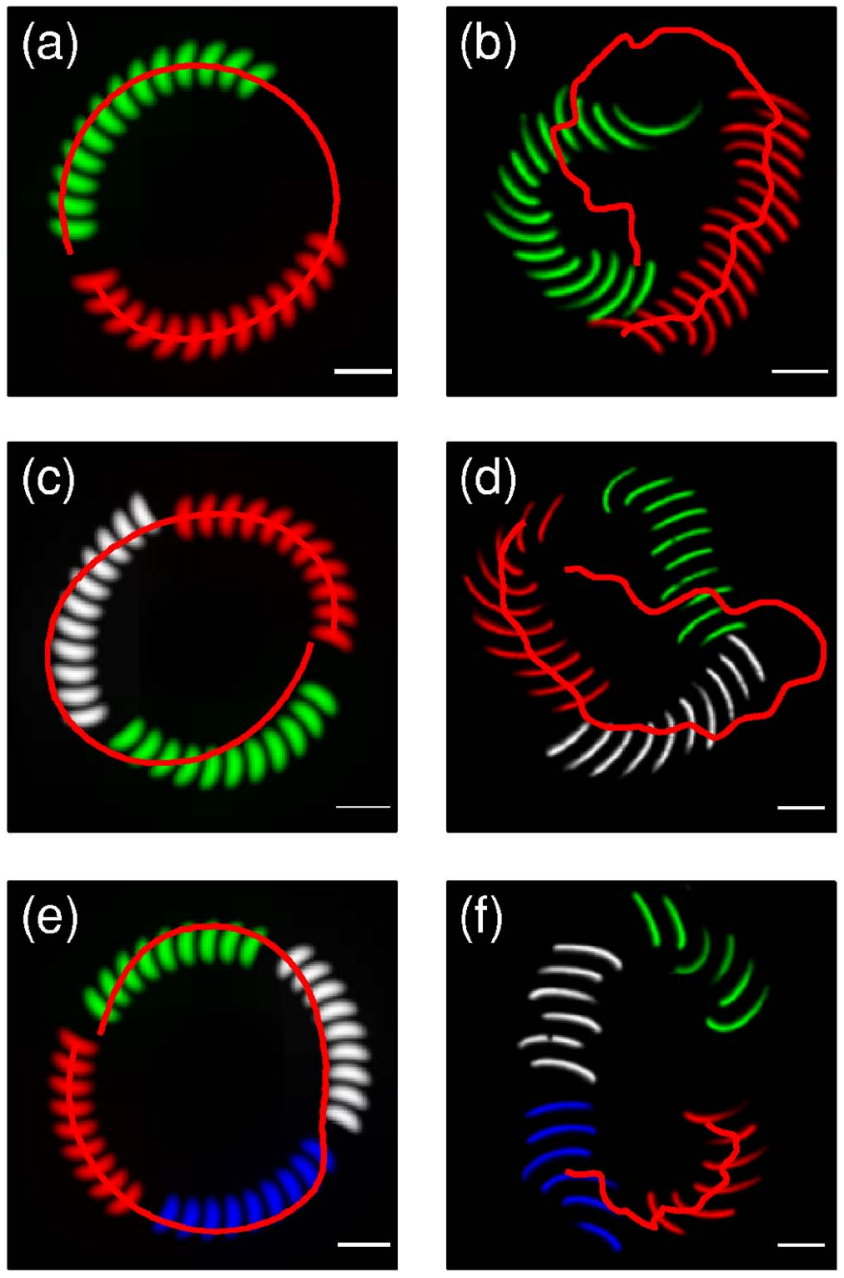

FIG. 6. (Color online) The left and right panels show a series of images of rotational waves in simulations and experiments, respectively. The waves are colored so they can be distinguished over the fraction of the period shown. The solid curve shows the trajectory of one wave over approximately one period, with the exception of panel (f). In panels (a) and (b), two waves rotate with respect to each other in an unstable orbit, with $c_{\text {sim }}=35$ and $c_{\text {exp }}=3.3 \times 10^{3} \mathrm{~mW} \mathrm{~cm}^{-2}$. In panels (c) and (d), three waves orbit in a stable configuration, with $c_{\text {sim }}=35$ and $c_{\text {exp }}=1.5 \times 10^{3} \mathrm{~mW} \mathrm{~cm}^{-2}$. In panels (e) and (f), four waves orbit in a stable configuration, with $c_{\text {sim }}=70$ and $c_{\text {exp }}=1.3 \times 10^{3} \mathrm{~mW} \mathrm{~cm}^{-2}$. The time between frames is $\sim 40 \mathrm{~s}$ in the experiments and 0.5 in the simulations. The other parameters and the scale bars are the same as in Fig. 5. The stability of the orbits in (a), (b) and (c), (d) is described in Fig. 7.

rotation, where the group centroid of the two waves spirals outward until the waves align in a processional mode. Figures $7(\mathrm{~d})-7(\mathrm{f})$ show a stable three-wave rigid rotation. The three-wave and four-wave rotational modes were perturbed by changing the position of one of the waves, and the altered configuration relaxed back to the original rotation following the small perturbation. Each wave in the three-wave configuration follows an apparent quasiperiodic trajectory, with the centroid of the system approximately following a circle. With increasing $c$, the circle decreases in size until it collapses to a noisy point. The individual wave orbits in the four-wave case have the features of a rounded square or superellipse, and the centroid of the system is localized at a noisy point. 

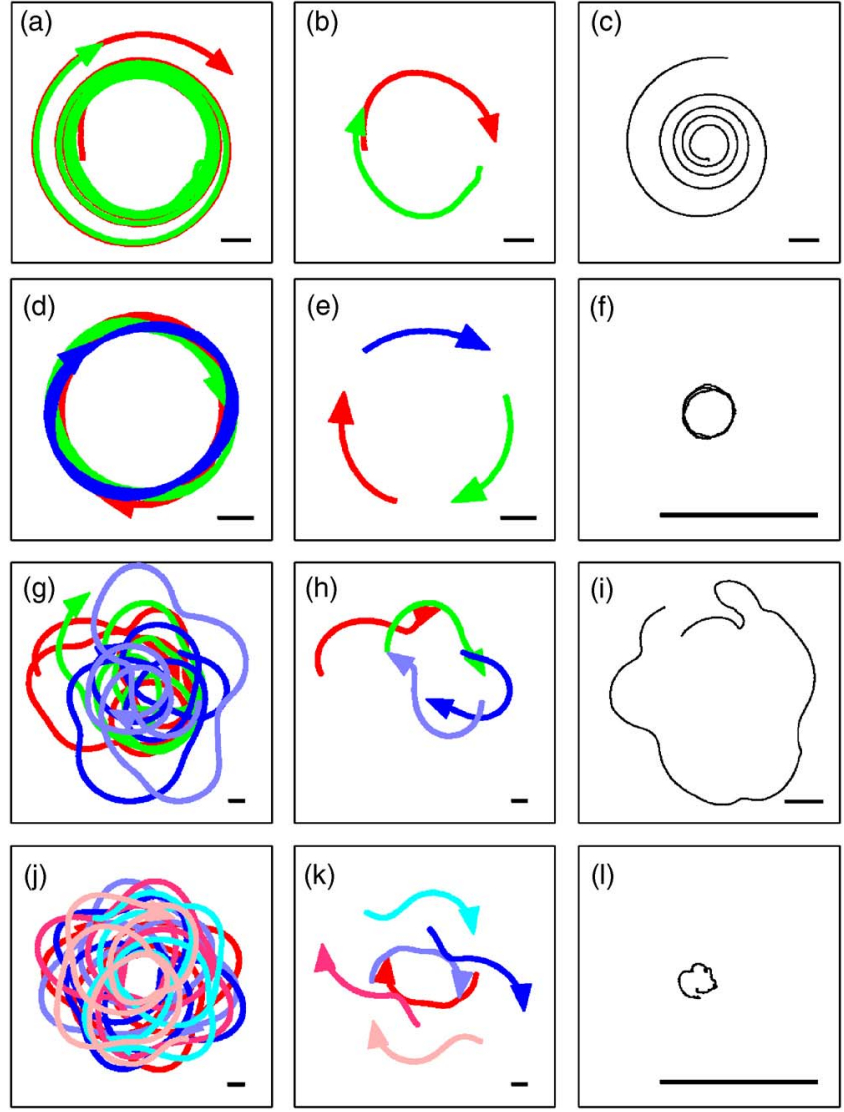

(I)

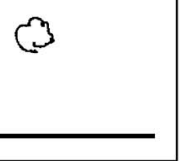

FIG. 7. (Color online) Examples of rotational behavior for various interaction strengths. The left-hand panels show the wave trajectories over a long period, $\sim 100$ dimensionless time units, while the middle panels show the trajectories over a short period, $\sim 10$ dimensionless time units. The righthand panels show the time evolution of the group centroid. Panels (a)-(c) show two waves in an unstable rotational mode, with $c_{\text {sim }}=35$. The group centroid spirals outward until the waves exhibit processional motion. Panels (d) - (f) show three waves in a rigid rotational mode, with $c_{\text {sim }}=70$, where the waves maintain a constant inter-wave spacing as they rotate. Panels (g)-(i) show a loose rotation of four waves, with $c_{\text {sim }}=12$, where the waves switch order as they rotate. An example of a more complex rotational structure is shown in panels (j)-(1). The six-wave rotation shows two three-wave subgroups orbiting each other, with $c_{\text {sim }}=8.0$. The length scale bar at the bottom of each panel is equal to $r_{0} / 4$. Other complex rotational structures are shown in Fig. 8.

\section{Other behaviors}

In addition to the simple rotational modes, we found a variety of complex rotational modes in our simulations. Some of the complex modes are shown in Figs. 7 and 8. Simple rotational modes were found for $N=2,3,4$ and complex rotational modes for $N=4,5,6,7,8$. The four-wave rotations shown in Figs. 6(e) and 6(f) and Figs. 7(g) and 7(h) and the six-wave rotations shown in Figs. $7(\mathrm{j})$ and $7(\mathrm{k})$ and Figs. 8(a) and 8(b) demonstrate that more than one rotational mode may occur with the same number of waves. Other behaviors include wandering and loose rotation. Wandering occurs at lower values of $c$, where the group moves in a processional manner but with a meandering group centroid. Loose rotation, in which the relative positions of the waves in a group shift, also occurs at lower values of $c$.

Figures $7(\mathrm{j})$ and $7(\mathrm{k})$ show an example of a complex rotation, with two groups of three waves orbiting around a


FIG. 8. (Color online) Examples of rotational modes with five, six, and eight waves. (a)-(c) Six waves form a stable structure, with one wave rotating in the middle of the other five, $c_{\mathrm{sim}}=32$. (d)-(f) Eight waves form a stable structure, with two waves rotating in the middle, $c_{\text {sim }}=75$. (g)-(i) Five waves form a stable structure, with three waves rotating in the middle, $c_{\text {sim }}=35$. See Fig. 7 for panel descriptions and Fig. 5 for other parameters.

common center. Additional shell-like complex rotations are shown in Fig. 8, where one, two and three waves rotating in the center are orbited by other waves in an outer shell configuration. These complex shell configurations develop from uniform initial conditions of waves equally spaced on a ring. Our numerical studies indicate that they are stable to small perturbations.

Rotational modes occur with each wave tracking a minimum in the excitability potential. In the case of a single wave, such as in the static LJ potential shown in Figs. 2(g) and 2(h), the wave remains near the path defined by $\nabla U=0$, with a slight offset for a finite turning rate. Interacting waves in multiple-wave rotations similarly track paths defined by $\nabla U=0$. A complex coupling arises, with each wave tracking a path of minimum potential resulting from the positions of the other waves as well as contributing to the path of minimum potential for each of the other waves.

\section{Parameters for group behaviors}

The various collective behaviors described above arise in small groups of interacting waves over specific ranges of the interaction strength $c$. At lower values of $c$, the waves behave in a noncohesive manner with interaction occurring only if the waves "collide," as shown in Fig. 4(a). The onset of cohesive behavior is indicated by a reduction in the average distance $D$ of the waves from the group centroid,

$$
D=\frac{1}{N} \sum_{n=1}^{N}\left|\mathbf{r}_{n}-\mathbf{r}_{g m}\right|,
$$

where 


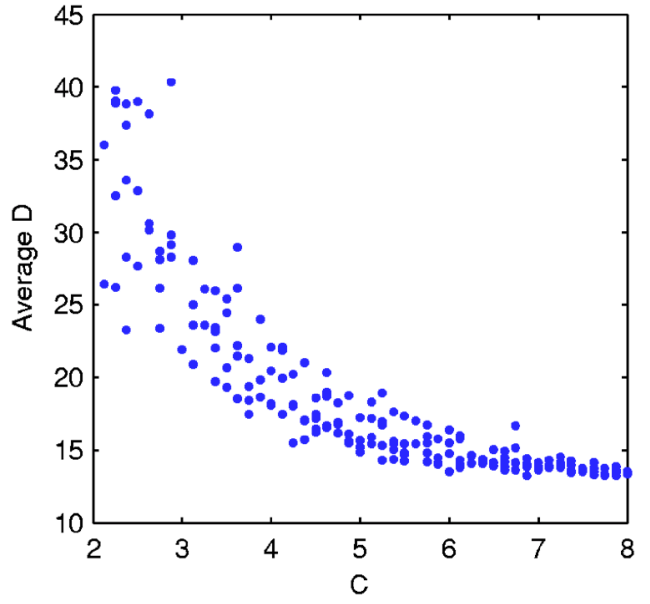

FIG. 9. (Color online) Average wave distance from group centroid as a function of the interaction strength $c$. Each point represents a different initial condition of the group of eight waves. As the strength of the interaction is increased, the mean wave separation from the group centroid decreases, representing a transition from noncohesive to cohesive group behavior. The simulations were carried out for 500 dimensionless time units for each initial condition, and the values of $D$ represent the time average of the wave distance following the decay of the transient behavior.

$$
\mathbf{r}_{g m}(t)=\frac{1}{N} \sum_{n=1}^{N} \mathbf{r}_{n}(t) .
$$

The value of $D$ is generally small for cohesive behavior, regardless of the details of the behavior, and large for noncohesive behavior. Figure 9 shows the time average of $D$ in an eight-wave system as a function of $c$ for varying initial conditions. For larger values of $c$, the value of $D$ remains small with little deviation, indicating cohesive behavior of the group. At lower values of $c$, the average value of $D$ is larger, and the individual values of $D$ depend upon the initial conditions. The value of $c$ at which $D$ becomes virtually independent of initial conditions was used to define the transition from noncohesive to cohesive behavior.

Figure 10 shows approximate regions of the various types of behavior as a function of the number of waves $N$ and the interaction strength $c$. The transition to coherent behavior is shown by the boundary between regions I and II. For values of $c$ above the transition, the group behaves cohesively, regardless of the initial conditions, while below the transition, the group may or may not behave cohesively, depending on the initial conditions. Stable processional motion occurs for all values of $c$ above the transition. Simple and complex rigid rotations generally occur at higher values of $c$, as shown in region III, and may be realized with initial conditions of waves equally spaced on a ring or by slowly changing $c$ to a target value from an existing rotational state. The limits for rotational behavior were found by tracking existing rotational states until they collapsed to a processional state. Initial conditions of waves equally spaced on a ring lead to loose rotation or wandering for values of $c$ slightly above the transition from noncohesive to cohesive behavior, shown by region II.

The coexistence of rotational and processional modes for a given value of $c$ is illustrated in Fig. 11 for a four-wave

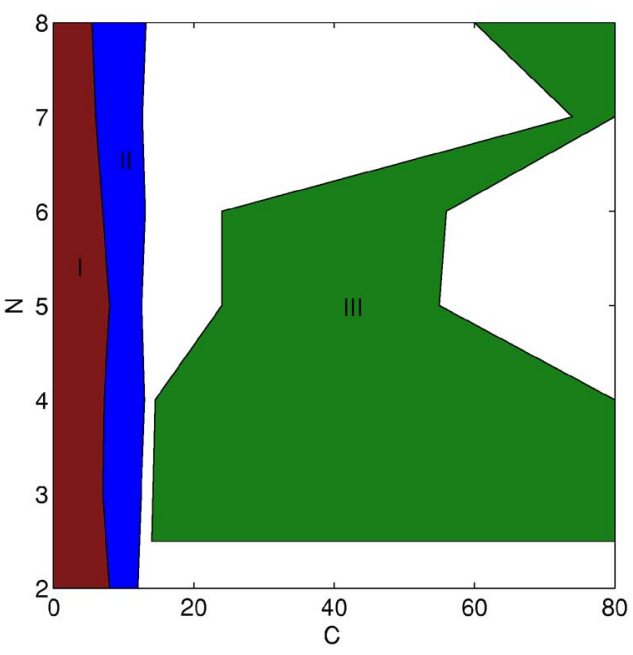

FIG. 10. (Color online) Approximate regions of different types of collective behavior as a function of the number of waves $N$ and the interaction strength $c$. Noncohesive motion occurs in region I; wandering and loose rotation occur in region II; and rigid rotation occurs in region III. Processional motion is observed for all values of $c$ above the boundary between region I and region II (blue, white, and green areas).

system. The two modes can be characterized by the average angular momentum $R$ around the group centroid and the mean velocity $P$,

$$
R=\frac{1}{N}\left|\sum_{n=1}^{N}\left(\mathbf{r}_{n}-\mathbf{r}_{g m}\right)\right| \times \hat{\mathbf{v}}_{n},
$$

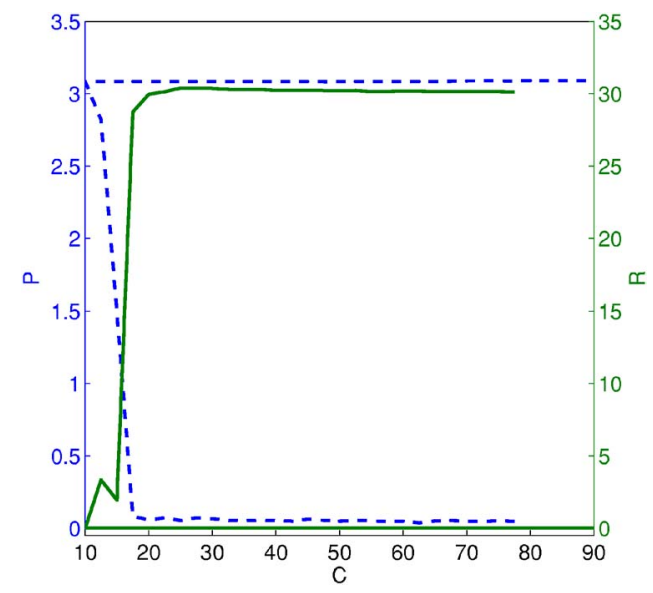

FIG. 11. (Color online) Processional and rotational group behavior as a function of the interaction strength $c$. Values of the group processional measure $P$ (dashed blue line) and rotational measure $R$ (solid green line) are given on the left-hand side and right-hand side, respectively. High values of $P$ or $R$ correspond to predominantly processional or rotational behavior, respectively. The $x$ axis gives the value of the interaction parameter $c$, which is first decremented and then incremented during the simulation. The fourwave simulation begins at a high value of $c$ with the group in a rotational mode. As the value of $c$ is decreased, the group remains in the rotational mode, indicated by the high value of $R$ and low value of $P$. A transition to parallel motion takes place with a reduction in the value of $R$ and an increase in the value of $P$. The value of $c$ is then increased. The group motion remains processional, indicated by the high value of $P$ and low value of $R$, as the value of $c$ is increased. 


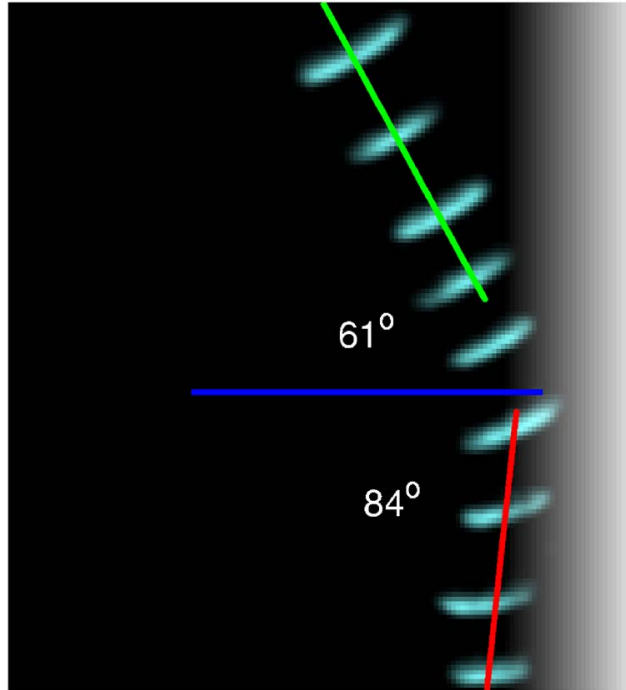

FIG. 12. (Color online) Superimposed experimental images showing a wave reflecting from a boundary. An asymmetry in the response to the potential gives rise to a larger reflection angle than the incidence angle. The boundary is defined by the linear potential $U(x)=2.3\left(x-x_{0}\right) \mathrm{mW} \mathrm{cm}^{-2} / \mathrm{mm}$, which is applied when the wave centroid crosses $x_{0}$. The corresponding linear intensity gradient is superimposed on the image.

$$
P=\frac{1}{N}\left|\sum_{n=1}^{N} \hat{\mathbf{v}}_{n}\right| .
$$

When the group is in a rotational or processional state, $R$ or $P$ will be large, respectively.

Starting in a rotational state at $c=80$, with large $R$ and $P$ near zero, the interaction strength is slowly reduced in a stepwise manner to $c=10$. The rotational state loses stability at $c \approx 20$, and the finite values of both statistics for $c$ between $\sim 10$ and $\sim 20$ result from a mixture of wandering and loose rotational behavior. Wandering gives rise to finite values of $R$ over short time periods; however, the sign of $R$ may change with the direction of the group, and the average value of $R$ is therefore small over long times. The system finds a processional state at $c \approx 10$. As the value of $c$ is increased, the system remains in the processional state with small $R$ and large $P$ values. This type of hysteresis has been previously termed "collective memory," 22 i.e., the group dynamics is dependent on the current set of interaction parameters as well as the group history.

\section{ASYMMETRIC WAVE RESPONSE TO EXCITABILITY GRADIENTS}

The damping of the wave motion in the harmonic valley potential shown in Figs. 2(a) and 2(b) indicates that there is an asymmetric response of the wave to the excitability gradient. Rather than oscillating around the minimum of the potential with a constant amplitude, the amplitude decreases as the wave progresses in the $x$ direction. The rate at which the wave turns is higher when it is traveling away from the potential minimum than when it is moving toward the minimum. The asymmetric response is also evident in the nonsymmetric reflection shown in Fig. 12. Experiments with periodic variations in excitability have shown that wave

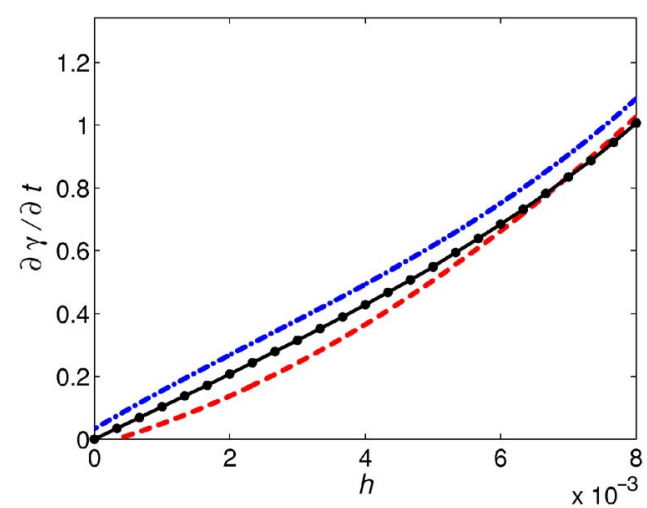

FIG. 13. (Color online) The dependence of the turning rate of a wave $\partial \gamma / \partial t$ on the variation of the light intensity gradient. The solid line shows the asymptotic values of $\partial \gamma / \partial t$ when a constant gradient $h$ is applied, where the wave orbits with a constant radius and a constant turning rate. With the gradient linearly increasing in time, $h=h_{\max } t / t_{\max }$, the wave turns at a higher rate (dot-dashed line) than the corresponding steady-state turning rate. With a gradient linearly decreasing in time, $h=h_{\max }\left(t_{\max }-t\right) / t_{\max }$, the wave turns at a lower rate (dashed line) than the steady-state turning rate. For the increasing and decreasing gradient, the constants are $t_{\max }=7.5$ and $h_{\max }=0.01$

segments grow or shrink with increasing or decreasing excitability, respectively, at approximately the same exponential rate. $^{23}$ However, because wave curvature decreases at the receding end and increases at the growing end of a wave segment, an asymmetry arises in the turning rate of a wave in an excitability gradient. This can be illustrated by subjecting a wave to an increasing or decreasing light intensity gradient and comparing the rates of turning in the different cases.

Consider a stabilized wave subject to a gradient governed by each of the following expressions:

$$
\begin{aligned}
& |\nabla U|=h, \\
& |\nabla U|=\frac{h_{\max } t}{t_{\max }}, \\
& |\nabla U|=\frac{h_{\max }\left(t_{\max }-t\right)}{t_{\max }},
\end{aligned}
$$

where $t_{\max }=7.5, h_{\max }=0.008$, and $0<t<t_{\max }$. The orientation of the gradient is maintained along $\hat{\mathbf{v}}_{\perp}$ as $t$ varies between 0 and $t_{\max }$. Equation (11) corresponds to a wave subjected to a constant turning gradient $h$. The wave trajectory follows a circle, with the radius dependent on the value of $h$. Based upon the radius, the turning rate $\dot{\gamma}$ is calculated, where $\gamma$ is the wave heading. This is the steady state turning rate, $\dot{\gamma}_{\mathrm{ss}}$, which is plotted in Fig. 13 for values of $h$ between 0 and $h_{\text {max }}$. Equation (12) corresponds to a wave initially traveling in a straight line that is then subjected to a linearly increasing gradient between 0 and $h_{\max }$. By measuring $\gamma$ at each time step, $\dot{\gamma}$ can be estimated at the current value of $h$. Equation (13) corresponds to a gradient linearly decreasing from $h_{\max }$ to 0 . For a short time prior to decreasing $|\nabla U|$, the wave is subjected to a constant gradient $h_{\max }$ so that $\dot{\gamma}_{t=0}=\dot{\gamma}_{\mathrm{ss}, h=h_{\max }}$. Figure 13 shows that the turning rate is higher when a wave experiences an increasing gradient than when it experiences a decreasing gradient. The value of $t_{\max }$ was chosen to be 
similar to the interval of time required for a wave to cross the center of the harmonic valley potential from its initial displacement in Fig. 2(a). For larger values of $t_{\max }$, curves (12) and (13) are expected to approach the steady state curve (11).

\section{CONCLUSIONS}

The behavior of stabilized waves subject to static and dynamic excitability potentials is dependent on two key components. The first is the existence of paths of minimum potential along which waves tend to align. The second is an asymmetry in the response of a wave to an applied excitability gradient. The coupling of these two components leads to a wide variety of emergent spatiotemporal behavior, including stable trajectories for single wave systems and stable processional and rotational modes for multiple interacting waves.

\section{ACKNOWLEDGMENTS}

We thank the National Science Foundation (CHE0415392) and the W. M. Keck Foundation for supporting this research.

${ }^{1}$ I. R. Epstein and K. Showalter, J. Phys. Chem. 100, 13132 (1996).

${ }^{2}$ F. Sagués and I. R. Epstein, Dalton Trans. 2003, 1201 (2003).

${ }^{3}$ A. S. Mikhailov and K. Showalter, Phys. Rep. 425, 79 (2006).

${ }^{4}$ P. B. Umbanhowar, F. Melo, and H. L. Swinney, Nature (London) 382, 793 (1996)
${ }^{5}$ C. Bizon, M. D. Shattuck, J. B. Swift, W. D. McCormick, and H. L. Swinney, Phys. Rev. Lett. 80, 57 (1998).

${ }^{6}$ C. Bizon, M. D. Shattuck, J. B. Swift, and H. L. Swinney, Phys. Rev. E 60, 4340 (1999).

${ }^{7}$ E. Ben-Jacob, I. Cohen, and H. Levine, Adv. Phys. 49, 395 (2000).

${ }^{8}$ J. Toner and Y. Tu, Phys. Rev. E 58, 4828 (1998).

${ }^{9}$ J. Buhl, D. J. T. Sumpter, I. D. Couzin, J. J. Hale, E. Despland, E. R. Miller, and S. J. Simpson, Science 312, 1402 (2006).

${ }^{10}$ D. J. Hoare, I. D. Couzin, J. G. J. Godin, and J. Krause, Anim. Behav. 67, 155 (2004).

${ }^{11}$ A. Zaikin and A. Zhabotisnky, Nature (London) 225, 535 (1970).

${ }^{12}$ L. Kuhnert, Nature (London) 319, 393 (1986).

${ }^{13}$ T. Sakurai, E. Mihaliuk, F. Chirila, and K. Showalter, Science 296, 2009 (2002).

${ }^{14}$ H. J. Krug, L. Pohlmann, and L. Kuhnert, J. Chem. Phys. 94, 4862 (1990).

${ }^{15}$ S. Kadar, T. Amemiya, and K. Showalter, J. Phys. Chem. A 101, 8200 (1997).

${ }^{16}$ J. H. Moore, C. C. Davis, and M. A. Coplan, Building Scientific Apparatus (Perseus, New York, 1991).

${ }^{17}$ E. Mihaliuk, T. Sakurai, F. Chirila, and K. Showalter, Faraday Discuss. 120, 383 (2001).

${ }^{18}$ E. Mihaliuk, T. Sakurai, F. Chirila, and K. Showalter, Phys. Rev. E 65, 065602 (2002).

${ }^{19}$ V. S. Zykov and K. Showalter, Phys. Rev. Lett. 94, 068302 (2005).

${ }^{20}$ The gray level image is converted to a binary image, with all pixels above an intensity threshold set to 1 and those below set to 0 . The centroid of the wave is then calculated based on the distribution of pixels assigned the value of 1 (Ref. 13)

${ }^{21}$ In order to maintain waves in a finite region, a constant gradient was applied perpendicular to the wave velocity when a wave crossed the boundary of the defined region (Ref. 13).

${ }^{22}$ I. D. Couzin, J. Krause, R. James, G. D. Ruxton, and N. R. Franks, J. Theor. Biol. 218, 1 (2002).

${ }^{23}$ I. Sendiña-Nadal, E. Mihaliuk, J. C. Wang, V. Pérez-Muñuzuri, and K. Showalter, Phys. Rev. Lett. 86, 1646 (2001). 